\title{
Drugging all RAS isoforms with one pocket
}

\author{
Dirk Kessler*, $^{\text {(D) }}$, Andreas Bergner ${ }^{1}$ (D), Jark Böttcher ${ }^{1}$ (D) Gerhard Fischer ${ }^{1}$ (D), Sandra \\ Döbel $^{1}$, Melanie Hinkel ${ }^{1}$ (D) Barbara Müllauer ${ }^{1}$, Alexander Weiss-Puxbaum ${ }^{1}$ \& Darryl B \\ McConnell**,1 (iD) \\ ${ }^{1}$ Discovery Research, Boehringer Ingelheim Regional Center Vienna GmbH \& Co KG, 1121 Vienna, Austria \\ *Author for correspondence: dirk.kessler@boehringer-ingelheim.com \\ **Author for correspondence: darryl.mcconnell@boehringer-ingelheim.com
}

Activating mutations in the three human RAS genes, KRAS, NRAS and HRAS, are among the most common oncogenic drivers in human cancers. Covalent KRAS ${ }^{G 12 C}$ inhibitors, which bind to the switch II pocket in the 'off state' of KRAS, represent the first direct KRAS drugs that entered human clinical trials. However, the remaining $85 \%$ of non-KRAS ${ }^{\mathrm{G} 12 \mathrm{C}}$-driven cancers remain undrugged as do NRAS and HRAS and no drugs targeting the 'on state' have been discovered so far. The switch I/II pocket is a second pocket for which the nanomolar inhibitor BI-2852 has been discovered. Here, we elucidate inhibitor binding modes in KRAS, NRAS and HRAS on and off and discuss future strategies to drug all RAS isoforms with this one pocket.

First draft submitted: 4 July 2020; Accepted for publication: 27 July 2020; Published online:

11 August 2020

Keywords: BI-2852 • GTPase • KRAS • small molecule inhibitors

The three human RAS genes KRAS, NRAS and HRAS encode for four highly homologous isoforms (NRAS, HRAS, KRAS4A and KRAS4B). These RAS gene products are all small GTPases that control the level of numerous cell signaling processes implicated in cell growth regulation [1]. Activating mutations in RAS, most commonly at codons 12, 13 and 61, dramatically increase cell signaling and are among the most common oncogenic drivers in human cancers [2]. KRAS is the most frequently mutated oncogene predominantly mutated at positions G12 and G13, with mutation rates of $86-96 \%$ in pancreatic cancers [3], 40-54\% in colorectal cancers [4] and 27-39\% in lung adenocarcinomas [5]. NRAS mutations are predominantly found in melanoma and hematological malignancies [6,7], while for $H R A S$, mutations in salivary gland and urinary tract cancers [8,9] are the most common. In addition to cancer, germline RAS mutations have been observed in RASopathies. Rare mutations in NRAS at codons 50 and 60 [10] and KRAS at positions 14, 58 and 153 [11] have been reported for Noonan syndrome. A KRAS P34R alteration has been observed for a patient with cardiofaciocutaneous syndrome [11] whereas Costello syndrome is driven by oncogenic HRAS mutations at positions 12 and 13 [12]. The members of the RAS protein family cycle through two different conformational states defined by differential binding to nucleotides which regulate the signaling. In the 'off' state $\left(\mathrm{RAS}_{\mathrm{Off}}\right)$, RAS proteins are bound to the nucleotide GDP, while in the 'on' state $\left(\mathrm{RAS}_{\mathrm{On}}\right)$ they are bound to the nucleotide GTP [13]. Activation from the GDP bound KRAS Off to the GTP (or nonhydrolysable analogs) bound KRAS $_{\text {On }}$ form is catalyzed by GEF such as SOS1 and SOS2 [14]. The $\gamma$-phosphate of GTP keeps two flexible loop regions, switch I and switch II [15] in a compact conformation that induces signal transduction through RAS binding to downstream effectors (e.g., CRAF, PI3K and RALGDS) and further activates GEF catalyzed nucleotide exchange through allosteric GTPase binding to GEFs (e.g., SOS). Hydrolysis of the $\gamma$-phosphate of GTP produces KRAS $_{\text {Off }}$ and causes a conformational change in the switch regions, leading to the formation of an inactive state which is unable to bind to effector molecules [16,17]. RAS itself has intrinsic, but weak GTPase activity that is catalyzed by GTPase activating proteins leading to its inactivation again. As RAS proteins functionally interact with GEFs, GTPase activating proteins and a number of effector proteins they possess a high degree of intrinsic plasticity that enable the formation and adaptation of binding pockets and interfaces to specifically adapt to these interaction partners (Supplementary Figure 1). Single point mutations in RAS proteins alter the specific dynamic of the protein and hence differentially influence various steps of the cycle, leading to increased equilibrium levels of KRAS $_{\text {On }}$ and ultimately, via downstream effector pathways, to amplified cell growth. 


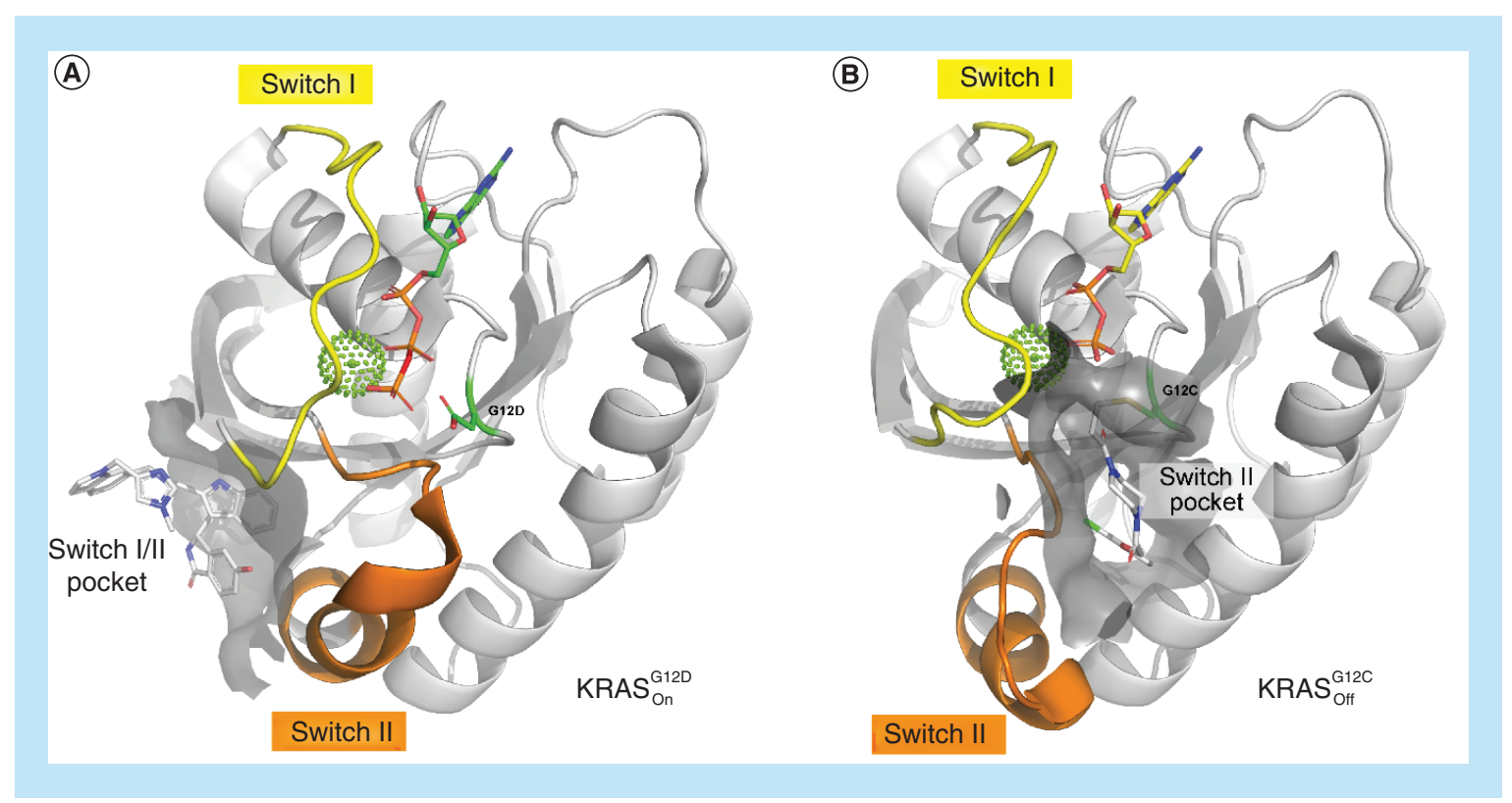

Figure 1. Switch regions and druggable sites of KRAS. Overview of the overall structure of KRAS highlighting the switch I (yellow) and switch II regions (orange). The respective switch pockets are shown with surface representation around the ligand-binding sites. (A) Ribbon plot of KRAS ${ }^{\mathrm{G} 12 \mathrm{D}}$ in the active conformation. The switch $\mathrm{I} / \mathrm{Il}$ region is highlighted around BI-2852 (PDB ID: 6GJ8). (B) Ribbon plot of KRAS ${ }^{G 12 C}$ in the inactive conformation with an acrylamide ligand covalently bound to amino acid G12C in the switch II pocket (PDB ID: 4M22). PDB: Protein Data Bank.

\section{Pros \& cons of RAS' two pockets}

The RAS family of proteins has been notoriously recalcitrant to drug discovery efforts since their discovery as oncogenes in the early 1980s [18] until the recent discovery of two potentially druggable pockets on RAS (Figure 1A $\&$ B). The Shokat group discovered a pocket on RAS positioned above the switch II loop (SII-pocket) using covalently linked small molecules $[17,19]$. The SII-pocket is closed in the active form of KRAS and can only be observed in inactive KRAS. Covalent inhibitors binding to the SII-pocket have successfully reached human clinical trials [20,21] but adaptive resistance mechanisms have already been elucidated [22]. Drugs targeting KRAS ${ }_{\text {Off }}$ are unlikely to be successful on KRAS mutants such as KRAS ${ }^{\mathrm{G} 12 \mathrm{R}}$ which do not cycle through the inactive form [23].

Another pocket that has been characterized is situated between the switch I and II regions (SI/II-pocket) of RAS, and is occupied by up to three distinct water molecules as seen in several apo $\mathrm{RAS}_{\mathrm{On}}$ crystal structures (PDB-Codes, e.g., 6QUU for KRAS ${ }^{\mathrm{G} 12 \mathrm{D}}$, 3OIW for HRAS ${ }^{\mathrm{G} 12 \mathrm{~V}}$ and 5UHV for NRAS ${ }^{\mathrm{wt}}$ ) (Figure 2B) with water \#1 being the most conserved and bound in all three RAS forms. In contrast, this is not observed in apo KRAS off protein crystal structures (e.g., 4LPK for KRAS ${ }^{\mathrm{wt}}$ or $6 \mathrm{ZIO}$ for NRAS ${ }^{\mathrm{wt}}$; Figure $2 \mathrm{~A}$ ). The SI/II-pocket is small and shallow having a volume and surface area of $12 \AA^{3}$ and $36 \AA^{2}$, respectively. Fragments binding to the SI/II-pocket were discovered by the Fesik group at Vanderbilt [24] and researchers at Genentech [25]. Recently, we published the first nanomolar chemical probe, BI-2852, which binds to the SI/II-pocket of all three RAS isoforms in both the on and off states [26].

The SI/II-pocket presents opportunities that are not accessible to the SII-pocket; develop drugs for each of the three isoforms KRAS, HRAS and NRAS as the region of the SI/II pocket is fully conserved (Supplementary Figure 2); target the on state of RAS to overcome adaptive resistance to KRAS $S_{\text {off }}$ drugs; and target the on state of RAS to address non-cycling RAS mutants. However, the SI/II-pocket also poses significant challenges. Sparring at least one wild-type RAS isoform is an expected requirement for a RAS drug, as triple RAS knockout mice embryos are not viable but can be rescued by reintroduction of an HRAS transgene [28]. Given that KRAS, HRAS and NRAS have identical sequences at the SI/II-pocket (Supplementary Figure 2), achieving sufficient RAS isoform selectivity poses a formidable hurdle. Also, the SI/II-pocket is a very small lipophilic pocket mainly formed by the key amino acids K5, V7, D54, L56, Y71 and T74. The lipophilic core is surrounded by a shallow polar rim (Figure 3) which will require precision design in order to achieve the potency required by drug molecules. In this work, we provide 

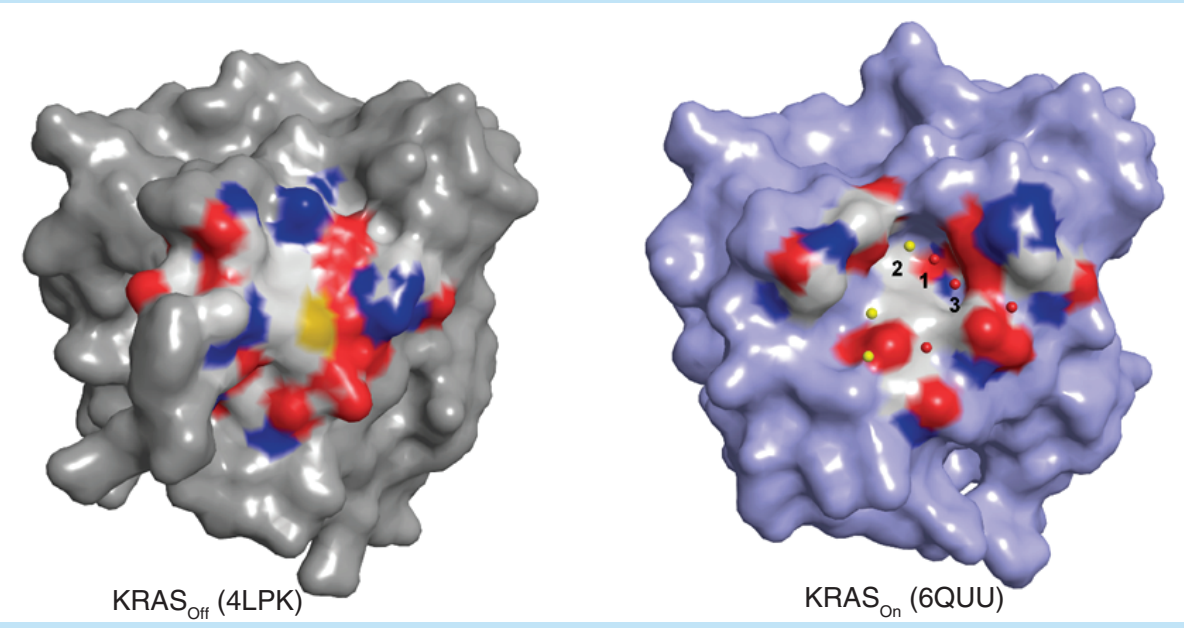

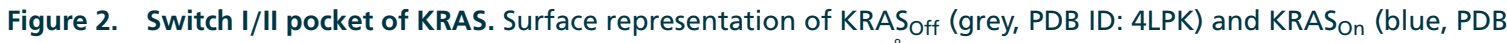
ID: 6QUU) with binding site crystallographic waters (cut-off radius $5 \AA$, waters are colored by their RISM free energy calculated with flare (water 'happiness'): yellow (indifferent waters): $-1.5<\Delta \mathrm{G}<1.5$; red (unhappy water): $\Delta \mathrm{G}>1.5$ (\#1: 2.16, \#2: 0.49, \#3: 2.68; all units in $\mathrm{kcal} / \mathrm{mol}$ ) around the switch I/II binding site [27]. The three most conserved waters are highlighted with numbers. There are no very 'happy' waters with highly negative $\Delta G$ values. PDB: Protein Data Bank; RISM: Reference interaction site model.

Figure 3. The lipophilic hot spot of switch I/II. $\mathrm{SI} / \mathrm{Il}$-pocket with the relevant crystallographic water molecules and amino acids in the small lipophilic pocket and the shallow polar rim surrounding the small cavity.

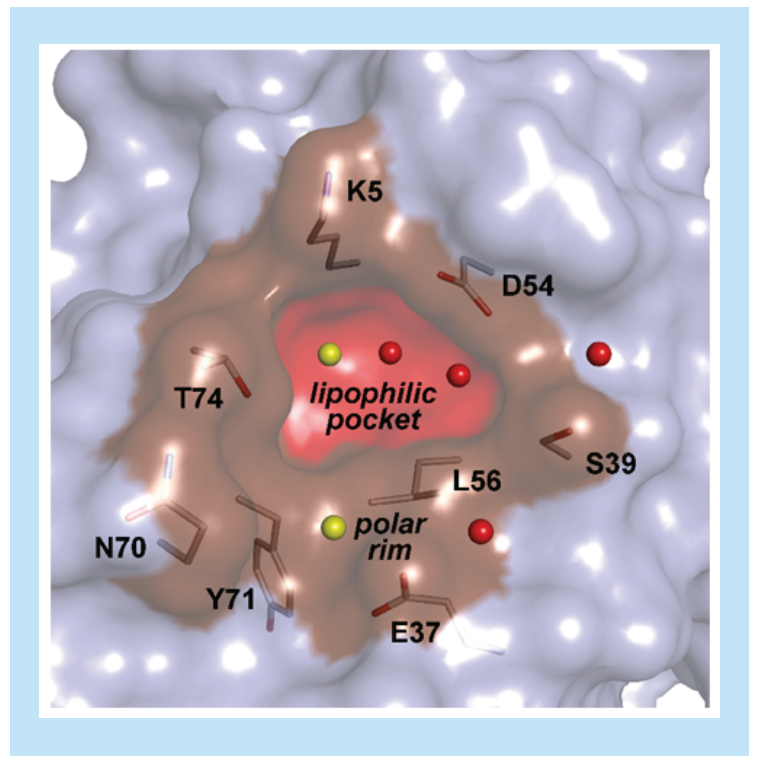

$\mathrm{x}$-ray crystal structures elucidating in detail how ligands bind to the SI/II-pocket in KRAS, NRAS and HRAS in both the on and off states. The establishment of robust cocrystallization systems [26] and high-throughput soaking systems [29] has allowed us to generate a high coverage of relevant RAS crystal structures and thus gain insights into designing more potent and specific SI/II-pocket inhibitors or proteolysis targeting chimeras (PROTACs) for the three RAS family of proteins. The high-throughput crystallization system also allowed us to develop our so called 'x-ray first' approach where we crystallized every newly synthesized compound in the active KRAS ${ }^{\text {G12D }}$ form before proceeding toward biophysical or biochemical affinity testing. Based on the binding mode we selected the interesting molecules for further measurements to neglect the typical affinity biased optimization strategies that often lead to wrong conclusions with respect to binding interactions. 


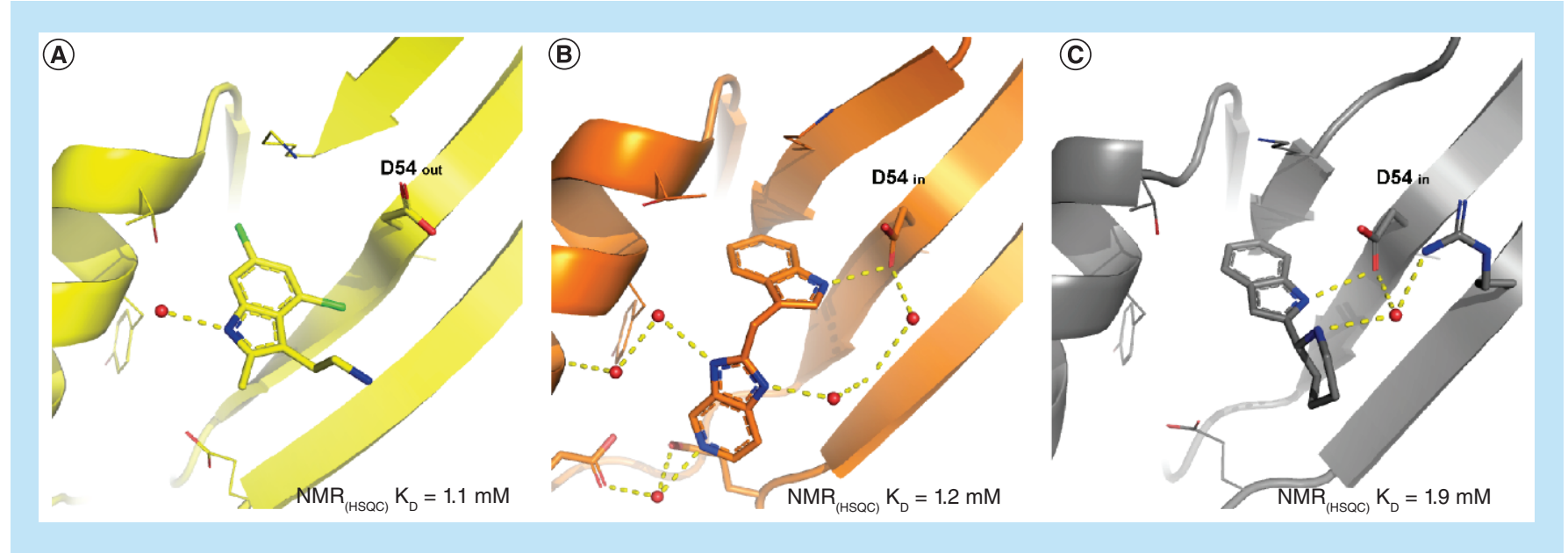

Figure 4. Indoles as privileged scaffold. Published indole starting points in the switch I/II of KRAS ${ }^{\mathrm{G} 12 \mathrm{D}}$ with respective NMR HSQC binding affinities. (A) Maurer et al. (4DST) [25], (B) Sun et al. (4EPV) [24], (C) Kessler et al. with compound 13 (6ZLI) [26]. HSQC: Heteronuclear single quantum coherence; NMR: Nuclear magnetic resonance.

\section{Indoles: a privileged scaffold for the SI/II-pocket}

Similarly to Maurer et al. and Sun et al., we also identified indole starting points with millimolar affinity, using our fragment-based screening approaches (Figure 4). The high propensity for indoles to bind to the SI/II-pocket is determined by two structural key elements. First, the bicyclic aromatic indole core forms $\mathrm{CH}-\pi$ interactions with the side-chains of K5 and L56 (Figure 5C). Second, the indole NH forms an H-bond with the carbonyl of D54, selecting the in position over the out position. Both positions have also been observed in the apo KRAS On structure. The privileged binding of indoles to the SI/II-pocket is also explained by the apo structure of KRAS $\mathrm{Off}$, where Tyr71 from switch II occupies the SI/II-pocket also forming H-bonds to D54 and CH- $\pi$ interactions with K5 and L56 (Figure 5B). Upon indole-binding Tyr71 is flipped toward the switch II pocket, turning the S2 helix by about $35^{\circ}$ and adopting a conformation similar to that observed in the apo KRAS On structure.

The indole-binding elements are maintained as the compound potency is optimized from the starting fragments, leading to compound $\mathbf{1 8}$ from Kessler et al. and BI-2852 itself (Figures 5A-E). Of note is the altered position of $\mathrm{K} 5$ in the structure of BI-2852 which forms four $\mathrm{CH}-\pi$ interactions with the ligand in contrast to only one $\mathrm{CH}-\pi$ interaction with the fragments and 18 (Figure 5E). The 2,4-dichloroindole (4DST) shows a flipped binding mode maintaining the $\mathrm{CH}-\pi$ interactions with $\mathrm{L} 56$ without the H-bond to D54, explaining its very weak potency. The additional charge-charge interaction formed between D54 and the amino group of compound 13 with an NMR $\mathrm{K}_{\mathrm{D}}$ of $1.9 \mathrm{mM}$ is an important additional interaction.

\section{Selectivity between active KRAS, HRAS \& NRAS}

Despite the amino acid sequences of KRAS, HRAS and NRAS being identical in the region of the SI/II-pocket, surprisingly moderate selectivity between RAS isoforms in the active state has been observed for BI-2852 and compound 18. All interactions established by the original fragments were maintained by BI-2852 and 18 across all three RAS isoforms, namely the $\mathrm{CH}-\pi$ interactions between the indole moiety and $\mathrm{K} 5$ and L56 and the H-bond and charge-charge interactions between D54.

While isothermal titration calorimetry (ITC) data clearly demonstrate that BI-2852 binds all three RAS isoforms, intriguingly it binds tenfold more strongly to active $\operatorname{KRAS}^{\mathrm{G} 12 \mathrm{D}}$ versus $\mathrm{KRAS}^{\mathrm{wt}}$ (740 nM vs $\left.7.5 \mu \mathrm{M}\right)$. We have been unsuccessful in obtaining crystal structures of BI-2852 in the active form of wild-type KRAS. However, an overlay of apo phosphoaminophosphonic acid guanylate ester (GNP)-bound KRAS ${ }^{\text {wt }}$ (6GOD) and apo phosphomethylphosphonic acid guanylate ester (GCP)-bound KRAS ${ }^{\mathrm{G} 12 \mathrm{D}}$ (6QUU) structures shows a subtle difference in the switch I conformation (Figure 6A) leading to E37 adopting a position in wild-type KRAS which is unable to form an H-bond to the phenyl group and clashes with the imidazole of BI-2852 (Figure 6B). This could explain the observed weaker activity for wild-type KRAS. 
(A)

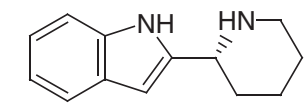

Compound 13

$\mathrm{NMR} \mathrm{K}_{\mathrm{D}} \mathrm{KRAS}_{\mathrm{On}}^{\mathrm{G} 12 \mathrm{D}}=1.9 \mathrm{M}$

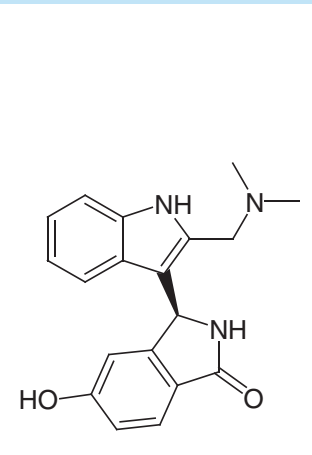

Compound 18

ITC $K_{D}$ KRAS $_{\text {On }}^{\mathrm{G} 12 \mathrm{D}}=22 \mu \mathrm{M}$

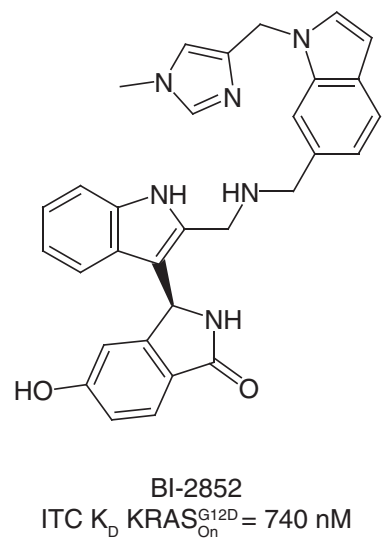

(B)

(B)

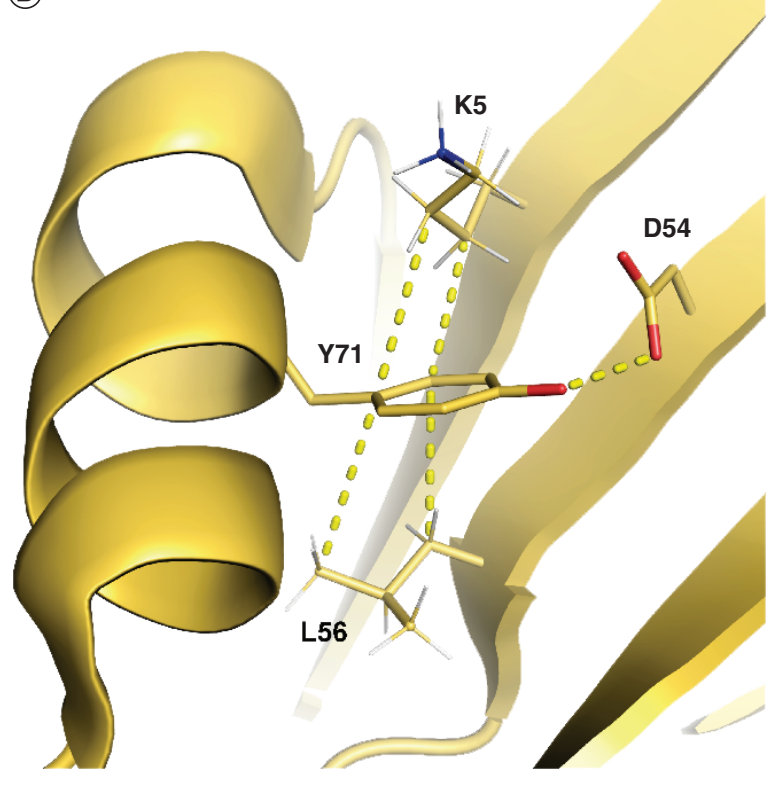

(D)

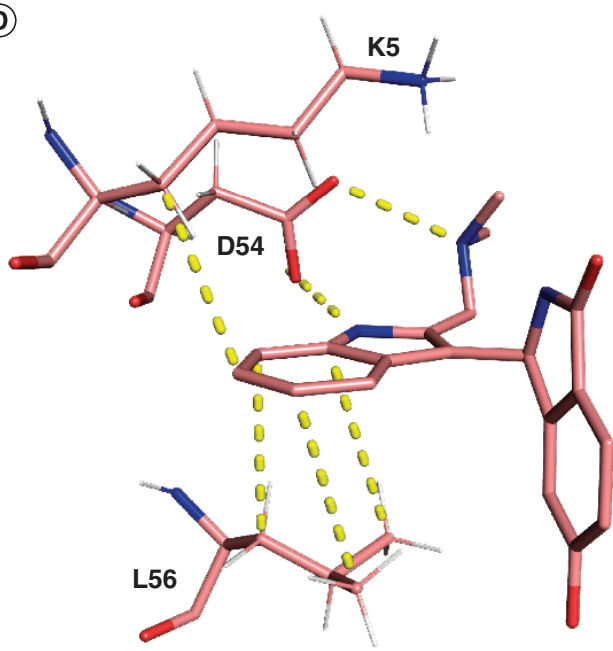

(C)

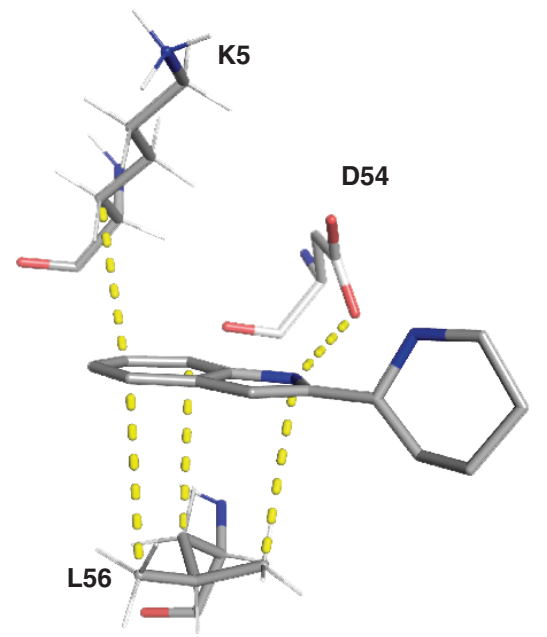

(E)

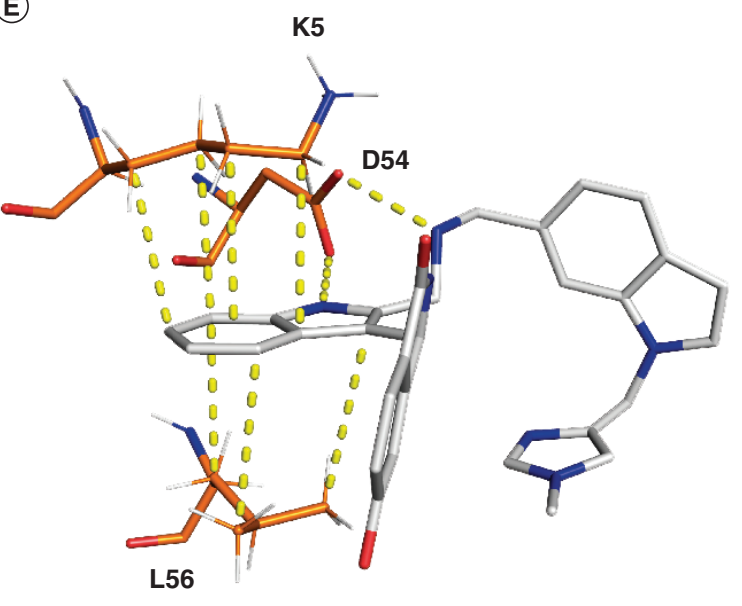

Figure 5. Optimization towards BI-2852. (A) Key compounds during optimization of $\mathrm{BI}-2852$ with respective $\mathrm{K}_{\mathrm{D}}$ values in active $K_{R A S}{ }^{G 12 D}$. (B) Shows the apo KRAS wt structure (4LPK), (C-E) shows key residues in the switch I/II pocket of active KRAS ${ }^{G 12 D}$ with 13 (6ZLI), 18 (6GJ6) and BI-2852 (6GJ8). Important $\mathrm{CH}-\pi$ interactions and $\mathrm{H}$-bonds are marked with dotted lines. 
(A)

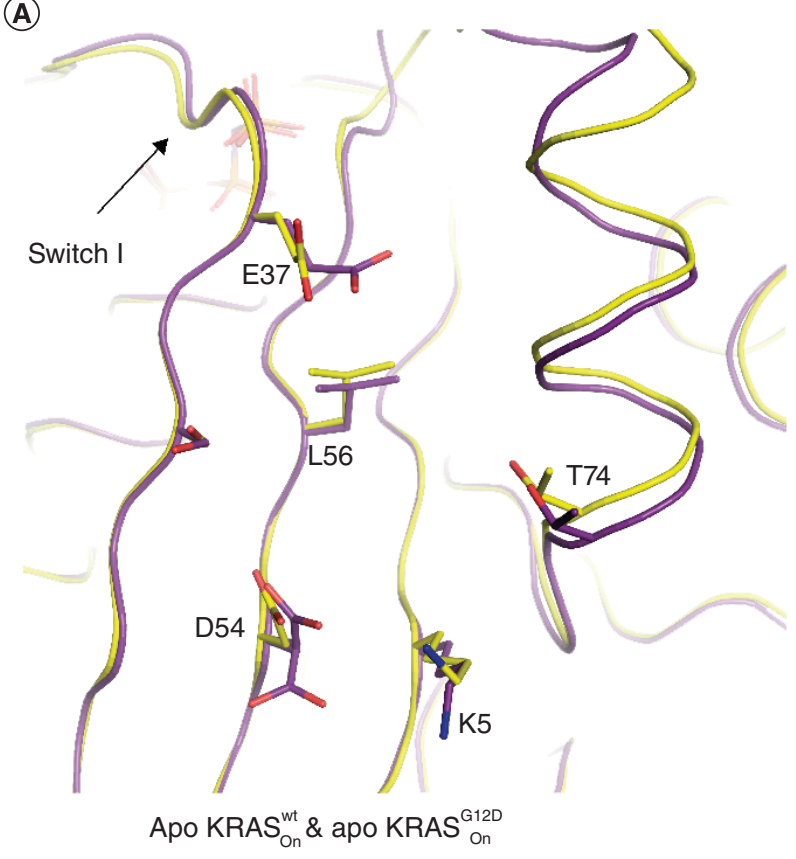

(B)

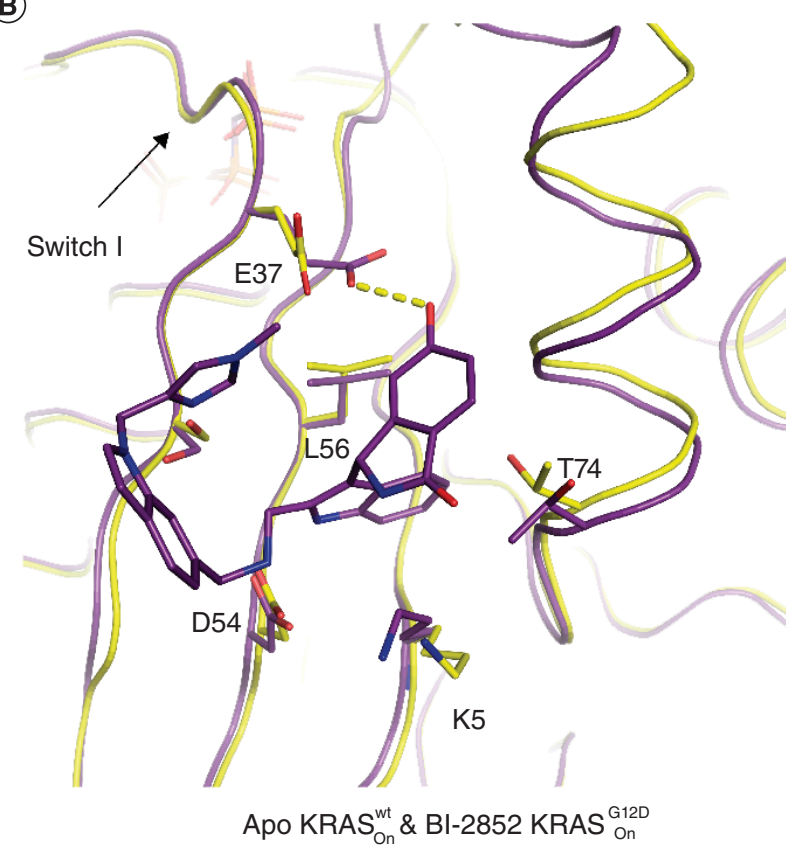

Figure 6. Apo versus ligand bound KRAS. Overlay of protein crystal structures of different KRAS variants in the active apo and ligand bound form. (A) Overlay of apo active KRAS ${ }^{w t}$ (yellow, 6GOD) and apo KRAS G12D (purple, 6QUU) with E37 in a conformation that does not accommodate binding of $\mathrm{BI}-2852$ (B) (active KRAS ${ }^{\mathrm{G} 12 \mathrm{D}}$ in purple, 6GJ8).

Intriguingly, the less decorated compound 18 displays a different selectivity profile compared with BI-2852, and binds most strongly to active HRAS ${ }^{\text {wt }}(6.7 \mu \mathrm{M})$, seven-times more weakly to active $\mathrm{KRAS}^{\mathrm{wt}}$ ( $49 \mu \mathrm{M}$ ), and exhibits intermediate binding affinity to active $\operatorname{KRAS}^{\mathrm{G} 12 \mathrm{D}}(22 \mu \mathrm{M})$ and active NRAS ${ }^{\mathrm{wt}}(33 \mu \mathrm{M})$. In order to elucidate the structural source of this selectivity profile we crystalized compound $\mathbf{1 8}$ into the active forms of KRAS, HRAS and NRAS utilizing crystallization systems of the strongly stabilizing oncogenic mutants KRAS ${ }^{\mathrm{G} 12 \mathrm{D}}, \mathrm{HRAS}^{\mathrm{G} 12 \mathrm{D}}$ and NRAS ${ }^{\text {61R }}$, respectively. In addition to the indole interactions, $\mathbf{1 8}$ forms two additional H-bonds with T74 and E37 in the active KRAS $^{\mathrm{G} 12 \mathrm{D}} \mathrm{x}$-ray crystal structure (Figure $7 \mathrm{~A}$ ) binding with a $\mathrm{K}_{\mathrm{D}}$ of $22 \mu \mathrm{M}$ and accounting for the approximate 50 -fold increase affinity from the original fragment hits. In active HRAS ${ }^{\mathrm{G} 12 \mathrm{D}}$ compound $\mathbf{1 8}$ forms all the interactions as observed in active KRAS ${ }^{\mathrm{G} 12 \mathrm{D}}$ but the iso-indolinone rotates slightly to stabilize a water molecule (water\#1) that is not observed in KRAS, most likely leading to the observed threefold increased $\mathrm{K}_{\mathrm{D}}$ of $6.7 \mu \mathrm{M}$ for active HRAS ${ }^{\text {wt }}$. To accommodate the rotation of $\mathbf{1 8}$, E37 moves slightly away maintaining the $\mathrm{H}$-bond to $\mathbf{1 8}$ but from the alternate carbonyl oxygen (Figure 7B). Binding of $\mathbf{1 8}$ to active GTP loaded NRAS ${ }^{\mathrm{Q} 61 \mathrm{R}}$ (Figure 7C), the weakest complex of the three isoforms, completely loses the $\mathrm{H}$-bond to T74, maintains bonding to water\#1 as in the GCP-bound HRAS ${ }^{\mathrm{G} 12 \mathrm{D}}$ structure, maintains the H-bond to E37 as in the GCP-bound KRAS ${ }^{\mathrm{G} 12 \mathrm{D}}$ structure, and finally forms an H-bond to a newly observed second water via the iso-indolinone NH. Of note is the GTP loaded nucleotide state of NRAS ${ }^{\mathrm{Q} 61 \mathrm{R}}$ without further need for a nucleotide exchange demonstrating the strong prevalence of KRAS $_{\text {On }}$ for that very specific mutation. These subtle differences in binding modes highlight both the potential for potency and selectivity optimization in the SI/II-pocket but also the precision of design required to achieve it. For design, the intrinsic plasticity of RAS proteins mentioned above (Supplementary Figure 1) constitutes both boon and bane. Here, the opening of the SI/II-pocket results in the binding of energetically unfavorable water molecules in the newly formed pocket, offering the replacement by other binding partners that is energetically favorable. This underpins the importance of ligand binding to water molecules, and more generally the potential of stringent incorporation of solvation analysis in compound design [30].

Precision of binding modes required to gain selectivity for one RAS isoform over another also needs to be maintained as molecules are grown to increase potency. This was not completely achieved growing from compound 

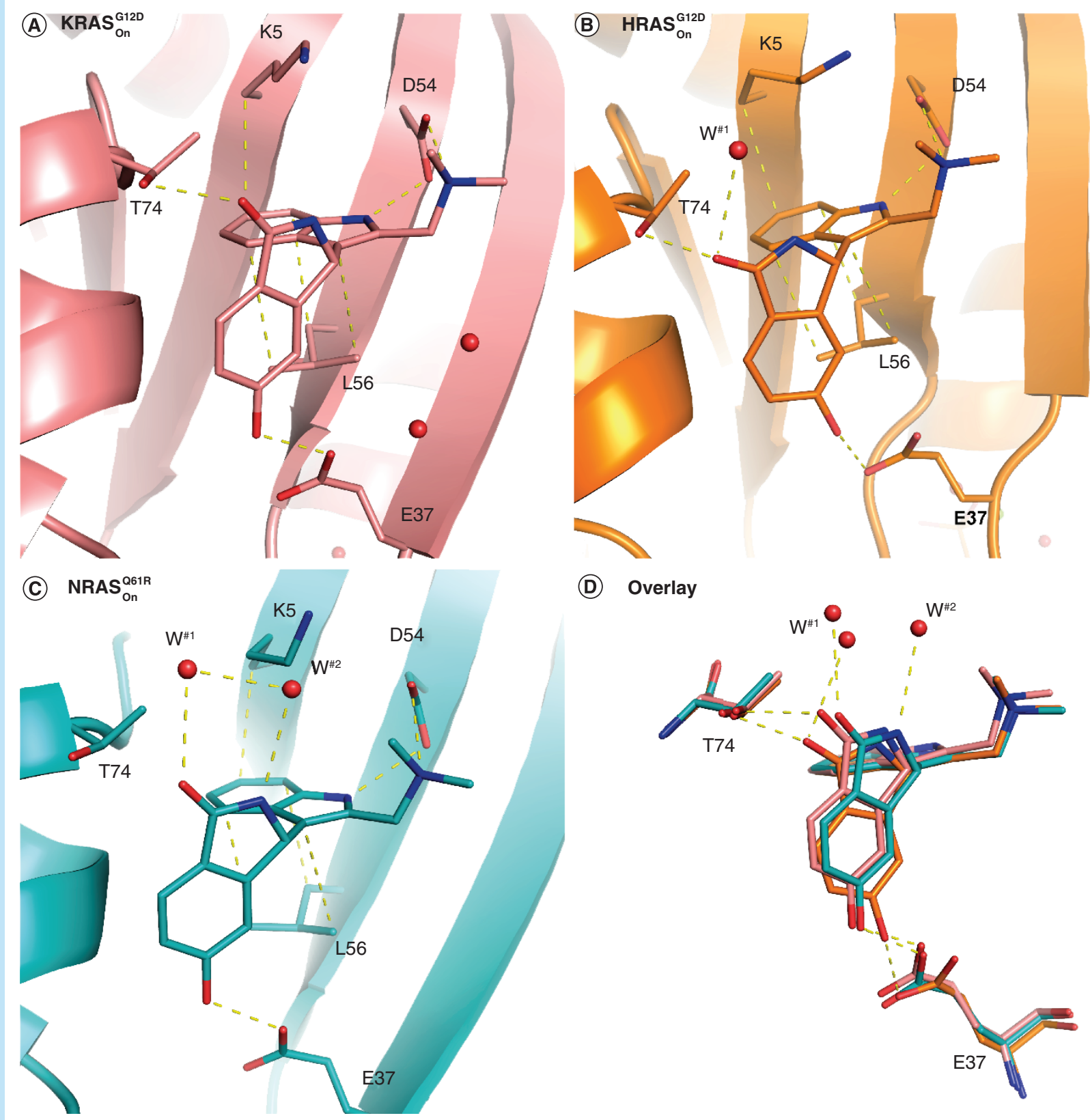

(D) Overlay

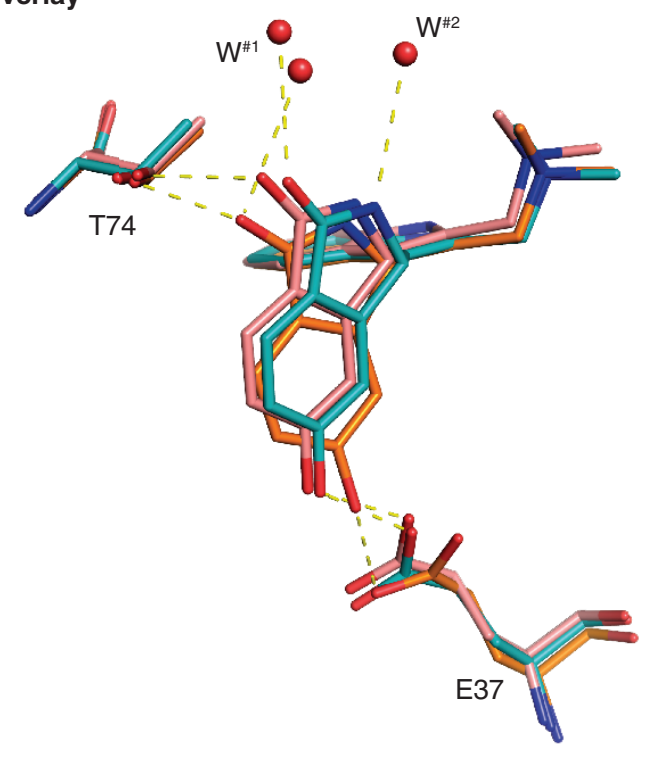

Figure 7. Compound 18 in different RAS mutant isoforms. Comparison of the binding mode of 18 in active RAS mutant forms. (A) Active $\operatorname{KRAS}^{\mathrm{G} 12 \mathrm{D}}$ (6GJ6), (B) active HRAS ${ }^{\mathrm{G} 12 \mathrm{D}}$ (6ZJ0), (C) active NRAS ${ }^{\mathrm{Q61R}}$ (6ZIZ) and (D) overlay of all three forms. Important waters in the binding pockets are highlighted with labels.

18 to BI-2852 whereby the H-bond to T74 was completely lost for KRAS ${ }^{\mathrm{G} 12 \mathrm{D}}$ (Figure 8) similarly as observed in the compound 18 NRAS ${ }^{\mathrm{Q} 61 \mathrm{R}}$ complex.

\section{Differences between the active \& inactive states}

In order to spare one of the wild-type isoforms of RAS, binding to both the active and inactive forms needs to

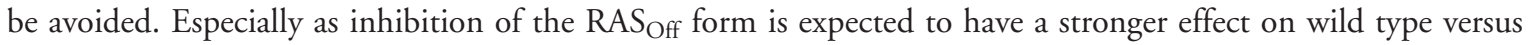
oncogenic mutant due to the former cycling faster through the GDP-bound state. Although $\mathbf{1 8}$ binds most strongly to the active form of HRAS ${ }^{\mathrm{G} 12 \mathrm{D}}$ it displays eightfold weaker binding to inactive $\operatorname{HRAS}^{\mathrm{wt}}$ (6.7 vs $\left.54 \mu \mathrm{M}\right)$. In 


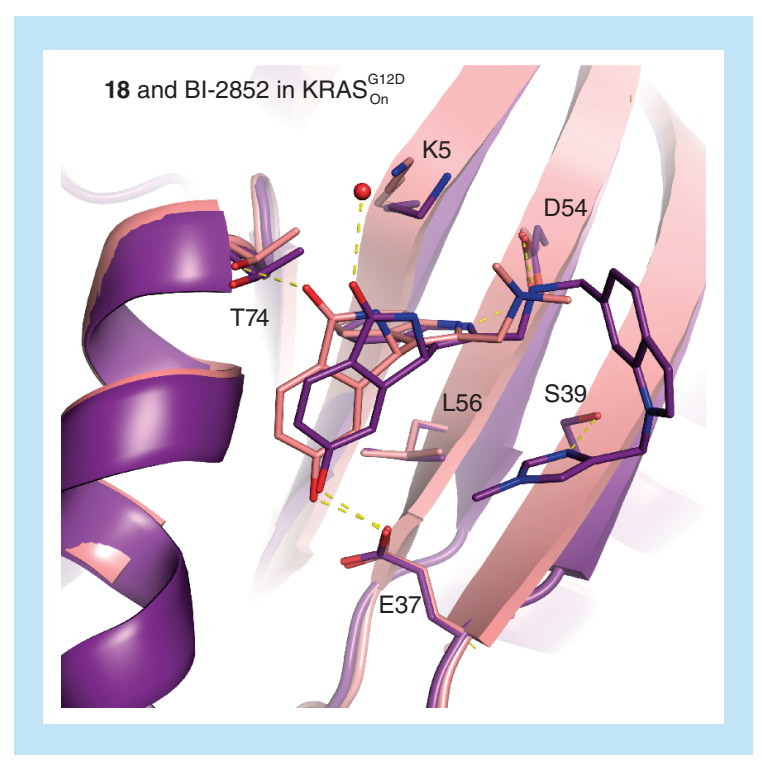

Figure 8. Comparison of 18 and BI-2852 in KRAS. Overlay of active KRAS ${ }^{\mathrm{G} 12 \mathrm{D}}$ with 18 (6GJ6) and $\mathrm{BI}-2852$ (6GJ8) demonstrating a conserved binding mode except for the lost H-bond of T74 with isoindolinone-oxygen when bound to $\mathrm{BI}-2852$.

order to understand this difference we determined the x-ray crystal structure of $\mathbf{1 8}$ and inactive HRAS ${ }^{\mathrm{wt}}$. There is a significant movement in switch I on going from the active to inactive form of HRAS resulting in a 2.5-3 $\AA$ displacement of E37. Despite this movement 18 still maintains the E37 H-bond albeit with the alternative carbonyl oxygen (Figure 9A \& B). The consequence of this is that $\mathbf{1 8}$ can no longer $\mathrm{H}$-bond to water \#1 which shifts slightly and forms an H-bond with T74 instead. Also, L56 in the off-state forms only two $\mathrm{CH}-\pi$ interactions with the indole of 18 versus the three interactions in the on-state. The loss of these two interactions accounts for the loss in binding affinity for inactive HRAS ${ }^{\mathrm{wt}}$. Compound 18 binds with the same affinity (ITC $K_{D}=33 \mu \mathrm{M}$ ) to the active and inactive states of NRAS ${ }^{\mathrm{wt}}$. In the binding mode of $\mathbf{1 8}$ in inactive NRAS ${ }^{\mathrm{wt}}$ (Figure 9C) the iso-indolinone is again rotated to accommodate the E37 movement on going from the active to inactive conformation but all interactions are maintained in both structures (Figure 9A), again in line with the observed affinities.

18 binds to all forms of KRAS, active and inactive $\mathrm{KRAS}^{\mathrm{G} 12 \mathrm{D}}$ and active and inactive $\mathrm{KRAS}^{\mathrm{wt}}$, with approximately equivalent ITC $K_{D}$ values of 22, 29, 49 and $15 \mu \mathrm{M}$, respectively. As we were unable to obtain x-ray crystal structures beyond GCP-KRAS ${ }^{\mathrm{G} 12 \mathrm{D}}$ (Figure 7A) we can only assume that the binding modes are the same. It is however important to obtain crystal structures for all forms when optimizing SI/II-pocket drugs as surprises can occur. Such is the case for $\mathrm{BI}-2852$ binding to active $\mathrm{KRAS}^{\mathrm{G} 12 \mathrm{D}}$ and inactive $\mathrm{KRAS}^{\mathrm{G} 12 \mathrm{D}}$ which also have very similar binding affinities, 0.74 and $2 \mu \mathrm{M}$, respectively, and could be assumed to have the same binding modes. While the ligand BI-2852 overlays in the active and inactive states of KRAS ${ }^{\mathrm{G} 12 \mathrm{D}}$, due to a large movement of the switch I loop in the GDP structure, E37 moves away from the SI/II-pocket losing the phenolic H-bond to BI-2852 which is replaced by water. Interestingly, a new $\mathrm{CH}-\pi$ interaction between the methylene of $\mathrm{D} 38$ and the $\pi$ system of the $N$-methyl imidazole group is formed regaining the lost affinity from the missing $\mathrm{H}$-bond to E37. (Figure 10).

\section{Future perspective}

Closely tracking compound binding modes when attempting to drug the RAS SI/II-pocket is crucial if structureactivity relationships are to be interpreted correctly. Subtle differences in binding mode across the RAS isoforms can lead to significant differences in ligand-binding affinities. Also compounds with similar binding affinities can adopt alternate binding modes. Ideally, crystal structures need to be obtained for all compounds synthesized which has now become possible with the advances in protein crystallography and given sufficiently robust and highthroughput crystallization systems. For the optimization of BI-2852, we applied an approach that we refer to as ' $\mathrm{x}$-ray first' whereby newly synthesized compounds were first co-crystallized in the active form of KRAS ${ }^{\mathrm{G} 12 \mathrm{D}}$ and based on the x-ray crystallographic data compounds were then chosen for affinity measurement. ' $\mathrm{x}$-ray first' avoids the compound selection bias which occurs with the classical compound profiling approach where affinity measurements are performed first and used to then select compounds for further profiling such as $\mathrm{x}$-ray crystallography. An $\mathrm{x}$ ray first approach will move drug design away from structure-activity relationships toward interaction-activity 

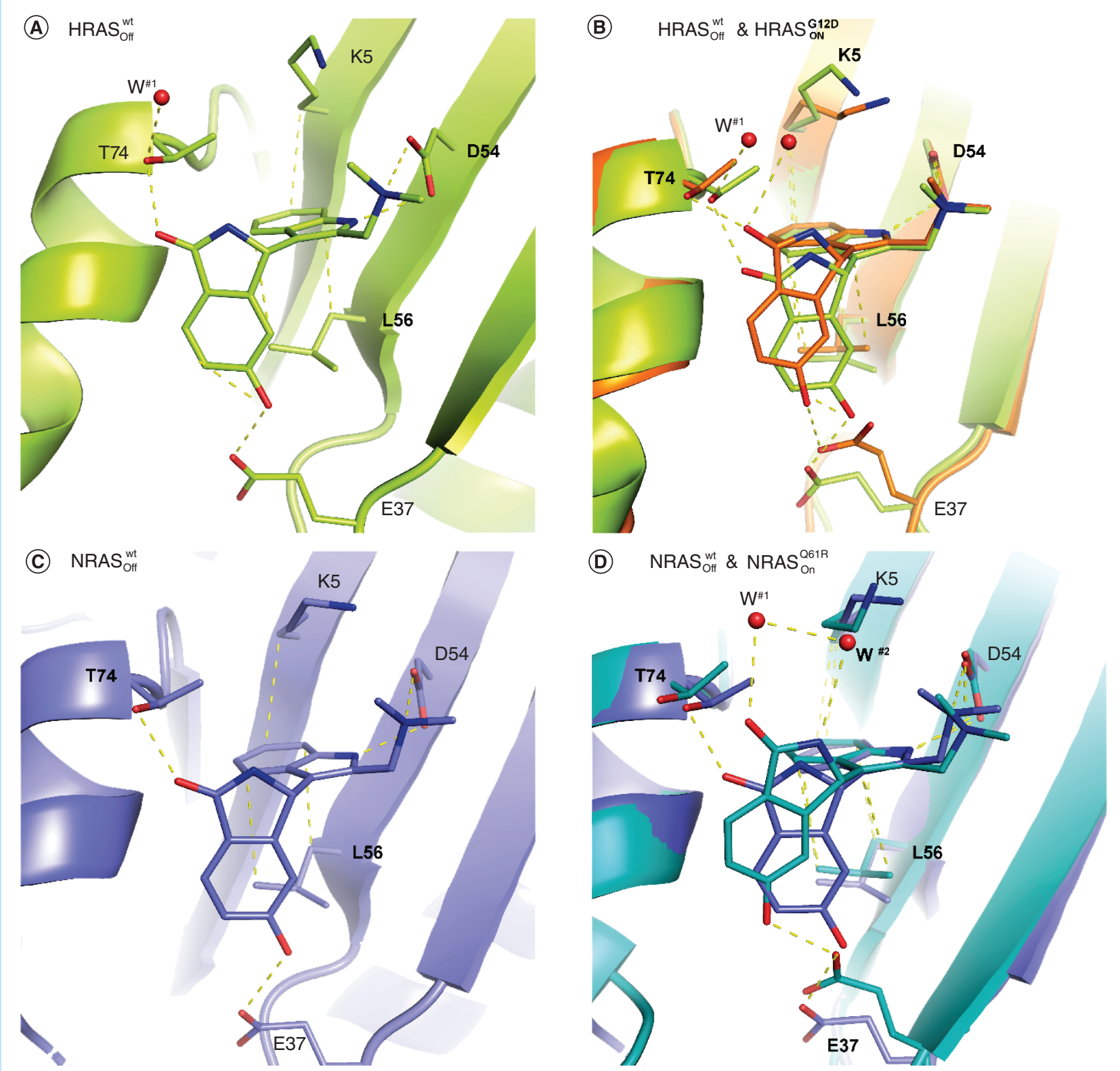

Figure 9. Differences in binding of $\mathbf{1 8}$ in active and inactive RAS forms. Comparison of the binding modes of $\mathbf{1 8}$ to (A) inactive $\operatorname{HRAS}^{\text {wt }}(6 Z I 3)$ and (B) inactive HRAS wt $(6 Z I 3)$ overlayed with active HRAS ${ }^{\mathrm{G} 12 \mathrm{D}}$ (6ZJ0) showing the adapted binding mode in the switch I/II pocket. (C) Shows the binding mode of 18 in inactive NRAS ${ }^{w t}(6 Z L 3)$ and (D) the overlay of inactive NRAS ${ }^{\text {wt }}$ (6ZL3) with active NRAS ${ }^{\text {Q61R }}$ (6ZIZ), again showing an adapted binding mode with E37 moving 2.5-3 $\AA$.

relationships which will be required to design molecules with the precision needed to drug challenging pockets such as the SI/II-pocket.

We have developed an approach that aggregates data from 3D structural ensembles into relevant hotspot information that can directly inform compound design and we could also demonstrate that non-obvious hidden opportunities can be found [31]. Given the distinct plasticity and dynamic nature of RAS proteins it is evident that a thorough understanding of RAS structures must be complemented by molecular dynamics approaches as a theoretical foundation. A number of papers, see for example, the work by Pantsar [32] and Gorfe et al. [33], have been published on this topic. However, bridging the gap between such analyses and their swift and direct application 


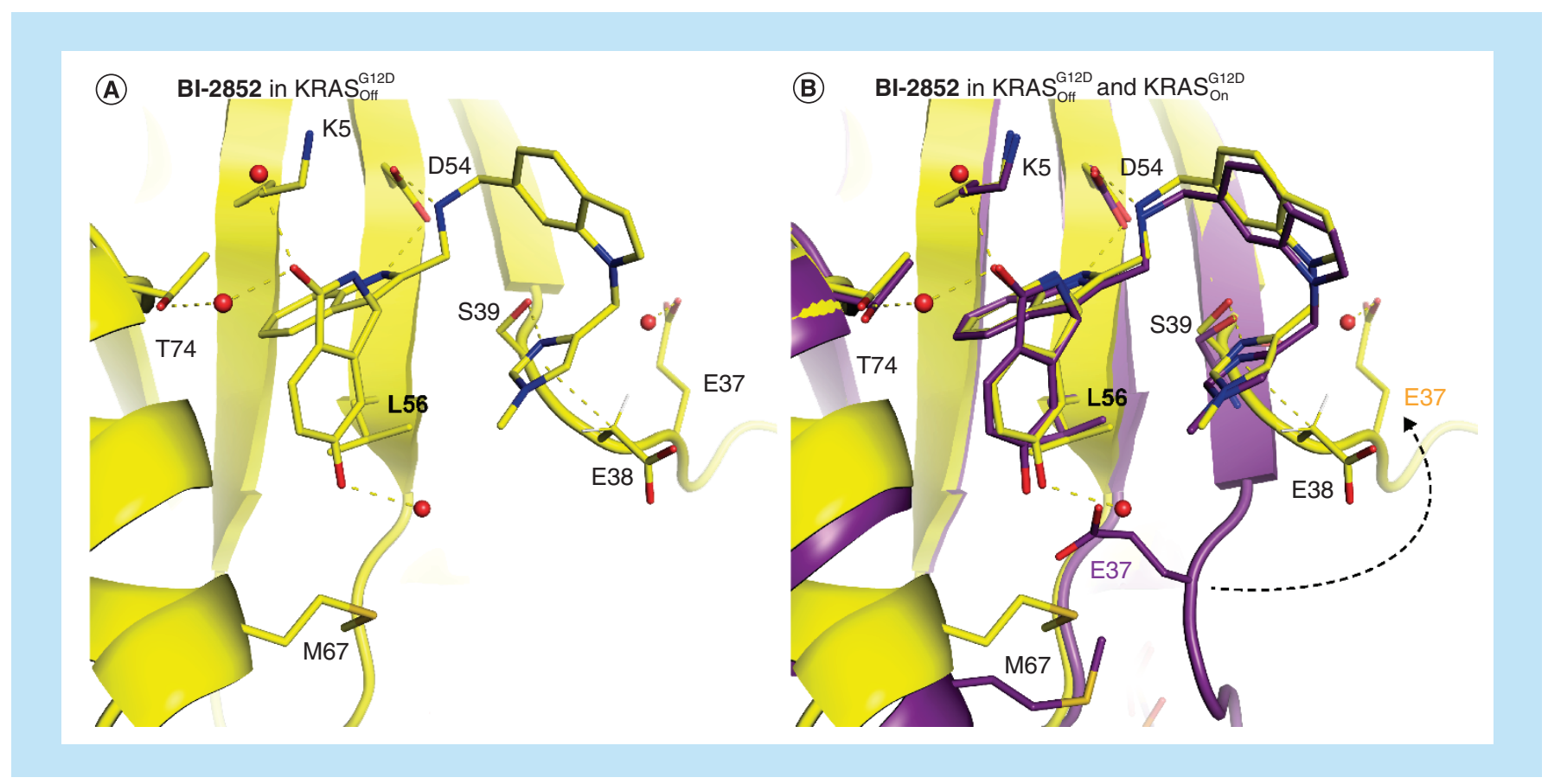

Figure 10. BI-2852 in inactive and active KRAS. BI-2852 bound to (A) inactive KRAS ${ }^{\text {12D }}$ (6ZL5) and (B) overlaid with BI-2852 in active KRAS $^{\mathrm{G} 12 \mathrm{D}}(6 \mathrm{GJ} 8)$.

in drug discovery remains a challenge, and is outside the scope of this paper. Our future efforts are committed toward vanishing this gap and seamlessly incorporating complex theory-driven approaches into our compound design processes.

It is interesting to note that all the subtly different binding modes for compound $\mathbf{1 8}$ arise from a rotation of the iso-indolinone with the privileged indole scaffold remain fixed in all structures. Structural rigidification of the bond between the indole and iso-indolinone resulting in natural product-like structures similar to the approach we took with mdm2 [34] should lead to an improvement of both potency and selectivity. It should be noted that all the observed SI/II-pocket interactions, including those with bound water molecules, have not yet been successfully addressed with one molecule. This highlights the potential for significant improvement beyond the current ligands but also the precision design challenge that this pocket poses.

The observation of protein-ligand interactions by $\mathrm{x}$-ray crystallography is clearly a powerful tool for drug design. However, the strength of individual interactions can only be inferred and not quantified. $\mathrm{CH}-\pi$ interactions between drug molecules and their target proteins, while often overlooked, are crucial determinants for their affinity. Indeed, $\mathrm{CH}-\pi$ interactions are the reason that indoles are a privileged structure for the SI/II-pocket. We have recently reported a new methodology, Pi by NMR [35], which can gauge the strength of individual $\mathrm{CH}-\pi$ interactions. We plan to extent the utility of this methodology and apply it to the SI/II-pocket which could identify scaffolds binding more tightly to the SI/II-pocket than indoles.

The challenge of drugging the SI/II-pocket has been significantly reduced with the advent of PROTACs which can substantially improve potency and selectivity of inhibitors [36]. Also, the first KRAS PROTACs utilizing covalent ligands binding to the SII-pocket have recently been described [37]. The insights discussed here can be equally applied to the optimization of SI/II-pocket binders and SI/II-pocket PROTACs. The SI/II-pocket clearly offers numerous opportunities to provide future medicines for RAS driven diseases particularly where an RAS $_{\mathrm{On}_{n}}$ drug is needed and for HRAS and NRAS driven disease for which no other options currently exist. 


\section{Author contributions}

D Kessler, J Böttcher and G Fischer designed the experiments, which were performed by S Döbel, M Hinkel, B Müllauer and A Weiss-Puxbaum. Data analysis were performed by D Kessler, J Böttcher, G Fischer and A Bergner performed the pocket plasticity and water analysis. D Kessler and DB McConnell drafted and edited the manuscript.

\section{Acknowledgments}

The authors would like to thank M Gmachl, A Mantoulidis, L Martin, M Mayer, A Gollner, B Wolkerstorfer and A Zöphel for their fundamental work in the project; I Vorwahlner, M Pearson, J Quant and N Kraut for their support, the opnMe.com team for providing the BI-2852 on the collaboration platform and A Waterson, J Phan and SW Fesik at Vanderbilt University, TN for the fruitful collaboration to target KRAS. We thank the staff at beamline X06SA at the Swiss Light Source, Paul Scherrer Institut, Villigen, Switzerland, for support and the Expose GmbH for crystallographic data collection.

\section{Financial \& competing interests disclosure}

This work was supported by the Austrian Forschungsförderungsgesellschaft (FFG) (grant nos. 854341, 861507, 867897 and 874517) ('Basisprogramme'). All authors are currently employees of Boehringer Ingelheim. The authors have no other relevant affiliations or financial involvement with any organization or entity with a financial interest in or financial conflict with the subject matter or materials discussed in the manuscript apart from those disclosed.

No writing assistance was utilized in the production of this manuscript.

\section{Open access}

This work is licensed under the Attribution-NonCommercial-NoDerivatives 4.0 Unported License. To view a copy of this license, visit http://creativecommons.org/licenses/by-nc-nd/4.0

\section{Executive summary}

\section{Overview}

- The three human RAS GTPases control numerous cell-signaling processes in cancer and RASopathies. Druggability of RAS

- The RAS family has two known pockets than can be drugged:

- The switch II pocket in inactive RAS has led to covalent inhibitors, now in clinical trials, for KRAS ${ }^{\mathrm{G} 12 C}$;

- The switch I/II pocket offers the possibility to develop inhibitors for all three RAS isoforms in both their active and inactive states. Attaining sufficient drug potency and selectivity in the switch I/II pocket is a significant challenge which will require high levels of structural information for drug design.

Indoles are a privileged scaffold of the SI/II pocket

- Two structural key elements of indoles are responsible for this preference:

- The aromatic indole core forms $\mathrm{CH}-\pi$ interactions with the side chains of $\mathrm{L} 56$ and $\mathrm{K} 5$;

- The indole $\mathrm{N}-\mathrm{H}$ forms an H-bond with the carbonyl of D54 leading to an 'Asp in' conformation.

Selectivity between KRAS, HRAS \& NRAS is achievable

- Biophysical and biochemical selectivity can be structurally explained with slightly differing binding modes of compound 18 and $\mathrm{BI}-2852$ in inactive and active RAS forms.

Future perspective

- Tracking compound binding modes by obtaining x-ray crystal structures for all compounds synthesized is critical when attempting to drug pockets as challenging as the $\mathrm{SI} / \mathrm{Il}$ pocket on RAS.

- Recent advances in 3D data analysis and molecular dynamics further help to understand the plasticity of RAS.

- Proteolysis targeting chimeras offer further possibilities to obtain sufficient potency and selectivity for RAS SI/II-pocket drugs.

- New modalities like proteolysis targeting chimeras are on the way that offer possibilities for more selective drugs for such difficult targets.

\section{References}

Papers of special note have been highlighted as: • of interest

1. Colicelli J. Human RAS superfamily proteins and related GTPases. Sci. STKE 2004(250), Re13 (2004).

2. Prior IA, Lewis PD, Mattos C. A Comprehensive survey of Ras mutations in cancer. Cancer Res. 72(10), 2457-2467 (2012).

3. Biankin AV, Waddell N, Kassahn KS et al. Pancreatic cancer genomes reveal aberrations in axon guidance pathway genes. Nature 491(7424), 399-405 (2012). 
4. Neumann J, Zeindl-Eberhart E, Kirchner T, Jung A. Frequency and type of KRAS mutations in routine diagnostic analysis of metastatic colorectal cancer. Pathol. Res. Pract. 205(12), 858-862 (2009).

5. Network CGAR. Comprehensive molecular profiling of lung adenocarcinoma. Nature 511(7511), 543-550 (2014).

6. Colombino M, Capone M, Lissia A et al. BRAF/NRAS mutation frequencies among primary tumors and metastases in patients with melanoma. J. Clin. Oncol. 30(20), 2522-2529 (2012).

7. Bacher U, Haferlach T, Schoch C, Kern W, Schnittger S. Implications of NRAS mutations in AML: a study of 2502 patients. Blood 107(10), 3847-3853 (2006).

8. Yoo J, Robinson RA. H-RAS gene mutations in salivary gland mucoepidermoid carcinomas. Cancer 88(3), 518-523 (2000).

9. Jebar AH, Hurst CD, Tomlinson DC, Johnston C, Taylor CF, Knowles MA. FGFR3 and RAS gene mutations are mutually exclusive genetic events in urothelial cell carcinoma. Oncogene 24(33), 5218-5225 (2005).

10. Cirstea IC, Kutsche K, Dvorsky R et al. A restricted spectrum of NRAS mutations causes Noonan Syndrome. Nat. Genet. 42(1), 27-29 (2010).

11. Schubbert S, Zenker M, Rowe SL et al. Germline KRAS mutations cause Noonan Syndrome. Nat. Genet. 38(3), 331-336 (2006).

12. Aoki Y, Niihori T, Kawame H et al. Germline mutations in HRAS proto-oncogene cause Costello syndrome. Nat. Genet. 37(10), 1038-1040 (2005).

13. Wittinghofer A, Pal EF. The structure of RAS protein: a model for a universal molecular switch. Trends Biochem. Sci. 16, 382-387 (1991).

- Reveals the structural function in detail for the first time.

14. Liceras-Boillos P, García-Navas R, Ginel-Picardo A et al. SOS1 disruption impairs cellular proliferation and viability through an increase in mitochondrial oxidative stress in primary MEFs. Oncogene 35(50), 6389-6402 (2016).

15. Milburn MV, Tong L, deVos AM et al. Molecular switch for signal transduction: structural differences between active and inactive forms of protooncogenic ras proteins. Science 247(4945), 939-945 (1990).

16. Vetter IR, Wittinghofer A. The guanine nucleotide-binding switch in three dimensions. Science 294(5545), 1299-1304 (2001).

17. Ostrem JM, Shokat KM. Direct small-molecule inhibitors of KRAS: from structural insights to mechanism-based design. Nat. Rev. Drug Discov. 15(11), 771 (2016).

18. Karnoub AE, Weinberg RA. Ras oncogenes: split personalities. Nat. Rev. Mol. Cell Biol 9(7), 517-531 (2008).

19. Ostrem JM, Peters U, Sos ML, Wells JA, Shokat KM. K-Ras (G12C) inhibitors allosterically control GTP affinity and effector interactions. Nature 503(7477), 548-551 (2013).

20. Canon J, Rex K, Saiki AY et al. The clinical KRAS (G12C) inhibitor AMG 510 drives anti-tumour immunity. Nature 575(7781), 217-223 (2019).

21. McCormick F. Sticking it to KRAS: covalent inhibitors enter the clinic. Cancer Cell 37(1), 3-4 (2020).

22. Xue JY, Zhao Y, Aronowitz J et al. Rapid non-uniform adaptation to conformation-specific KRAS (G12C) inhibition. Nature 577(7790), 421-425 (2020).

23. Hunter JC, Manandhar A, Carrasco MA, Gurbani D, Gondi S, Westover KD. Biochemical and structural analysis of common cancer-associated KRAS mutations. Mol. Cancer Res. 13(9), 1325-1335 (2015).

24. Sun Q, Burke JP, Phan J et al. Discovery of small molecules that bind to K-Ras and inhibit SOS-mediated activation. Angew. Chem. Int. 51(25), 6140-6143 (2012).

25. Maurer T, Garrenton LS, Oh A et al. Small-molecule ligands bind to a distinct pocket in Ras and inhibit SOS-mediated nucleotide exchange activity. Proc. Natl Acad. Sci. USA 109(14), 5299-5304 (2012).

26. Kessler D, Gmachl M, Mantoulidis A et al. Drugging an undruggable pocket on KRAS. Proc. Natl Acad. Sci. USA 116(32), 15823-15829 (2019).

27. Cheeseright T, Mackey M, Rose S, Vinter A. Molecular field extrema as descriptors of biological activity: definition and validation. $J$. Chem. Inf. Model 46(2), 665-676 (2006).

28. Nakamura K, Ichise $\mathrm{H}$, Nakao $\mathrm{K}$ et al. Partial functional overlap of the three RAS genes in mouse embryonic development. Oncogene 27(21), 2961 (2008).

29. Bergner A, Cockcroft X, Fischer G et al. KRAS binders hidden in nature. Chem. Eur. J. 25(52), 12037-12041 (2019).

30. Klebe G. Applying thermodynamic profiling in lead finding and optimization. Nat. Rev. Drug Discov. 14(2), 95-110 (2015).

31. Schmalhorst PS, Bergner A. A grid map based approach to identify nonobvious ligand design opportunities in 3D protein structure ensembles. J. Chem. Inf. Model 60(4), 2178-2188 (2020).

- Offers new technologies to further approach difficult targets like KRAS.

32. Pantsar T. The current understanding of KRAS protein structure and dynamics. Comput. Struct. Biotechnol. J. 18, 189-198 (2020)

33. Gorfe AA, Grant BJ, McCammon JA. Mapping the nucleotide and isoform-dependent structural and dynamical features of RAS proteins. Structure 16(6), 885-896 (2008). 
34. Gollner A, Weinstabl H, Fuchs JE et al. Targeted synthesis of complex spiro [ $3 H$-indole-3,2'-pyrrolidin]-2(1H)-ones by intramolecular cyclization of azomethine ylides: highly potent MDM2-p53 inhibitors. ChemMedChem 14(1), 88-93 (2019).

35. Platzer G, Mayer M, Beier A et al. PI by NMR: probing $\mathrm{CH}-\pi$ interactions in protein-ligand complexes by NMR. Angew. Chem. Int. Ed.

- Offers new technologies to further approach difficult targets like KRAS.

36. Farnaby W, Koegl M, Roy MJ et al. BAF complex vulnerabilities in cancer demonstrated via structure-based PROTAC design. Nat. Chem. Biol. 15(7), 672-680 (2019).

37. Zeng M, Xiong Y, Safaee N et al. Exploring targeted degradation strategy for oncogenic KRAS ${ }^{\mathrm{G} 12 \mathrm{C}}$. Cell Chem. Biol 27(1), 19-31 e16 (2020). 